\title{
Pancastatin A and B Have Selective Cytotoxicity on Glucose-Deprived PANC-1 Human Pancreatic Cancer Cells
}

\author{
Hae-Ryong Park* \\ School of Bioconvergence, Kyungnam University, Changwon 51767, Republic of Korea
}

Glucose deprivation and hypoxia frequently occur in solid tumor cells, including pancreatic cancer cells. Glucose deprivation activates the unfolded protein response (UPR) and causes the upregulation of glucose-regulated protein 78 (GRP78). Induction of GRP78 has been shown to protect cancer cells. Therefore, shutting down of GRP78 expression may be a novel strategy in anticancer drug development. Based on this understanding, a screening system established for anticancer agents that exhibit selective cytotoxicity on pancreatic cancer cells under glucose-deprived conditions. To test this hypothesis, the new compounds isolated, pancastatin A (PST-A) and B (PSTB), from Ponciri Fructus. PST-A and B were identified as glabretal triterpenoid moieties by electrospray ionization mass spectrometry and nuclear magnetic resonance spectroscopic methods. PST-A and B suppressed the accumulation of the UPR hallmark gene, GRP78, during glucose deprivation. Furthermore, PST-A and B showed selective cytotoxicity on PANC-1 pancreatic cancer cells under glucose deprivation. Interestingly, PST-A and B had no effect on these cells under normal growth conditions. Our results suggest that PST-A and B act as novel therapeutic agents to induce selective cell death in glucose-deprived pancreatic cancer cells.

Keywords: Pancastatin A, pancastatin B, glucose deprivation, GRP78, pancreatic cancer

Received: February 3, 2020 Accepted: April 8, 2020

First published online: April 9, 2020

*Corresponding author Phone: +82-55-249-2689 Fax: +82-505-999-2104 E-mail: parkhy@kyungnam.ac.kr

pISSN 1017-7825 elSSN 1738-8872

Copyright(C) 2020 by The Korean Society for Microbiology and Biotechnology

\section{Introduction}

Pancreatic cancer is one of the most serious, aggressive, and deadliest form of malignancies. Ductal adenocarcinoma of the pancreas, which account for $90 \%$ of all human pancreatic cancers, are an extremely lethal disease with an overall 5-year survival rate of approximately 5\% [1-3]. Pancreatic cancer is characterized by its resistance to almost all standard chemotherapy regimens due to the tolerance of cells to apoptosis. Therefore, there is a need to develop effective novel anticancer agents that have specific activity against pancreatic cancer cells. Hence, I focused on singularity of pancreatic cancer, a representative solid tumor, to establish a molecular target for the development of selective chemotherapeutic drugs [4-6]

Pancreatic cancer cells are characterized by their survival in an extreme environment of glucose deprivation, hypoxia, and nutrient deprivation $[7,8]$. Glucose deprivation is a physiological stress condition that is induced in several human diseases, such as ischemia and cancer [9]. This stress condition leads to the accumulation of unfolded or misfolded proteins in the lumen of endoplasmic reticulum (ER) in eukaryotic cells [10, 11]. The accumulation of unfolded proteins in the ER activates the unfolded protein response (UPR), which enhances cell survival by limiting the accumulation of unfolded or misfolded proteins in the ER [12]. Tumor cells need to adapt to the severe microenvironmental conditions, and induction of UPR is an important system in this response. UPR can be induced by glucose deprivation and the expression of glucose-regulated protein 78 (GRP78, also known as $\mathrm{BiP)}$ [12]. GRP78, also referred to as the immunoglobulin heavy chain binding protein, has been demonstrated to play a role in protecting tumor cells against intracellular-mediated cytotoxicity and against the toxic effects of anticancer agents $[13,14]$. GRP78 binds to the luminal domains of IRE1, ATF6, and PERK to maintain them within the ER, and activates these signaling pathways $[15,16]$. Upregulation of GRP78 expression in solid tumors protects tumor cells against various stresses. Thus, substances that directly down-regulate GRP78 expression might potentially be of use in the treatment of solid tumors, which are averse to successful cancer chemotherapy.

Based on this understanding, a novel screening system established to discover anticancer agents that process selective cytotoxicity against human pancreatic cancer cells under glucose deprivation. I recently screened 440 herbal medicines, and ensuring their stability, isolated two active compounds ensured stability, JS311-1 and 2, from Ponciri Fructus. By examining their structures and physicochemical properties, they were identified to possess glabretal triterpenoid moieties, and have not been previously reported as new compounds. These new compounds were designated as pancastatin A (PST-A) and B (PST-B).

In this study was investigated the effect of PST-A and B on GRP78 expression in pancreatic cancer cells exposed 
to glucose deprivation. Furthermore, it demonstrated the selective cytotoxicity of PST-A and B towards glucosedeprived PANC-1 human pancreatic cancer cells.

\section{Materials and Methods}

Isolation and Purification of Pancastatin A (PST-A) and B (PST-B)

Ponciri Fructus was obtained from Kumkang Pharm Co. Ltd., Changwon, South Korea. The dried Ponciri Fructus $(1.8 \mathrm{~kg}$ ) was extracted using $20 \mathrm{~L}$ of methanol for three days at room temperature and filtered through Whatman filter paper No. 1 (Advantec, Japan). Then, the methanol was removed by evaporation in vacuo, and a dried methanol extract was obtained. The resulting aqueous solution was extracted with ethyl acetate. The active material was applied to a silica gel column and eluted using $\mathrm{CHCl}_{3}: \mathrm{CH}_{3} \mathrm{OH}(100: 1$ to 1:1 [v:v]). Each fractions was subjected to analysis using thin layer chromatography (TLC). Selective cytotoxic activity of each fraction against the PANC-1 cells was then evaluated, and 50:1 fraction was found to be the most effective in inducing selective cytotoxicity under glucose-deprived conditions. The 50:1 fraction was applied on a Sephadex LH-20 column and eluted with $\mathrm{CH}_{3} \mathrm{OH}$. The active components that were eluted were purified by ODS sep-pak cartridge, using $80 \%$ $\mathrm{CH}_{3} \mathrm{OH}$. To separate the major compound, high performance liquid chromatography (HITACHI L-2130, Japan) was performed in an ODS column with $\mathrm{CH}_{3} \mathrm{OH}-\mathrm{H}_{2} \mathrm{O}(85: 15[\mathrm{v} / \mathrm{v}])$ as the eluent. Active compounds were obtained as a single peak detected at $267 \mathrm{~nm}$. Repeated HPLC finally gave two active compounds, JS-311-1 and JS-311-2. The structures of JS311-1 and 2 were determined using electrospray ionization mass spectrometry (Shimadzu LCMS-IT-TOF, Japan) and nuclear magnetic resonance (JEOL JNM-ECA600) spectroscopic methods. From the comparison of database and literature searches, we found that JS311-1 and 2 were novel compounds, and JS311-1 and 2 were designated as pancastatin A (PST-A) and B (PST-B), respectively (Fig. 1) [17].

\section{Cell Culture and Treatments}

PANC-1 human pancreatic adenocarcinoma cells were obtained from the Korea Cell Line Bank (Republic of Korea). The cells were maintained in DMEM medium (Gibco BRL, USA) supplemented with $10 \%$ heat-incubated fetal bovine serum (FBS; Hyclone, USA), penicillin (100 U/ml), streptomycin $(100 \mu \mathrm{g} / \mathrm{ml})$ and $3.7 \mathrm{mg} / \mathrm{ml} \mathrm{NaHCO}$. PANC-1 cells were cultured in a $37^{\circ} \mathrm{C}$ in humidified atmosphere containing $5 \% \mathrm{CO}_{2}$. To induce glucose deprivation, added a chemical stress, 2-deoxyglucose (2DG). 2DG as a chemical stressor is a substance such as hypoglycemia-mimicking agent $[11,12]$. 2DG was purchased from Sigma (USA) and dissolved in sterilized distilled water at a stock concentration of $2 \mathrm{M}$. 2DG was added to the culture medium at a final concentration of $20 \mathrm{mM}$.

\section{Observation of Morphological Changes}

PANC-1 cells in DMEM medium containing $10 \%$ FBS were seeded in 6-well plates $\left(2.0 \times 10^{5}\right.$ cells $\left./ \mathrm{ml}\right)$ and incubated at $37^{\circ} \mathrm{C}$ with $5 \% \mathrm{CO}_{2}$ for $48 \mathrm{~h}$. The cells were treated with various concentrations of PST-A and B. After 30 min incubation, 2DG was added to the well with a final concentration of $20 \mathrm{mM}$, and the plates were reincubated. After incubation for $24 \mathrm{~h}$, the cellular morphology was observed using an inverted microscope (Nikon, Japan) at $100 \times$ magnification.

\section{Colony Formation Assay}

PANC-1 cells were seeded at $1 \times 10^{5}$ cells $/ \mathrm{ml}$ in 24-well plates and, incubated for $48 \mathrm{~h}$ in a humidified atmosphere of $5 \% \mathrm{CO}_{2}$ at $37^{\circ} \mathrm{C}$. Cells were pretreated with various concentrations of PST-A and B. After $30 \mathrm{~min}$ incubation, 2DG was added to the wells a final concentration of $20 \mathrm{mM}$, and the plates were reincubated for $24 \mathrm{~h}$. For the colony formation assay, the cells were then diluted in new medium, replated at $1.0 \times 10^{3} \mathrm{cell} / \mathrm{s} / \mathrm{ml}$ per well in 6-well plates, and cultured for 7 to 8 days at $37^{\circ} \mathrm{C}$ in a humidified atmosphere containing $5 \% \mathrm{CO}_{2}$. Formed colonies were fixed with $10 \%$ formaldehyde, stained with $0.01 \%$ crystal violet, and counted. Cell survival (mean values with $95 \%$ confidence intervals from triplicate determinations) was calculated by setting the survival of control cells as $100 \%$. $\mathrm{IC}_{50}$ values (concentration required for $50 \%$ inhibition of colony formation) were determined from the dose-response curves of colony formation inhibition.

\section{Western Blotting Analysis}

Whole cell lysates were prepared by solubilizing cell in $1 \times$ sodium dodecyl sulfate (SDS) sample buffer (62.5 mM Tris-HCl, pH 6.8, 2\% SDS, 5\% 2-mercaptoethanol, and 10\% glycerol) as described. Equal amounts of

A

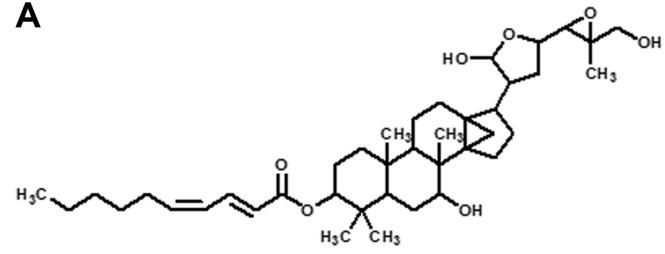

Pancastatin A (PST-A)

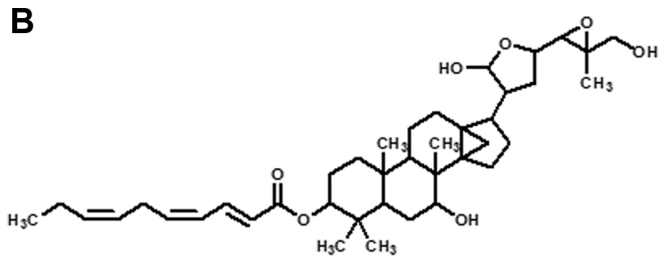

Pancastatin B (PST-B)

Fig. 1. Chemical structures of Pancastatin A (PST-A) and B (PST-B). 


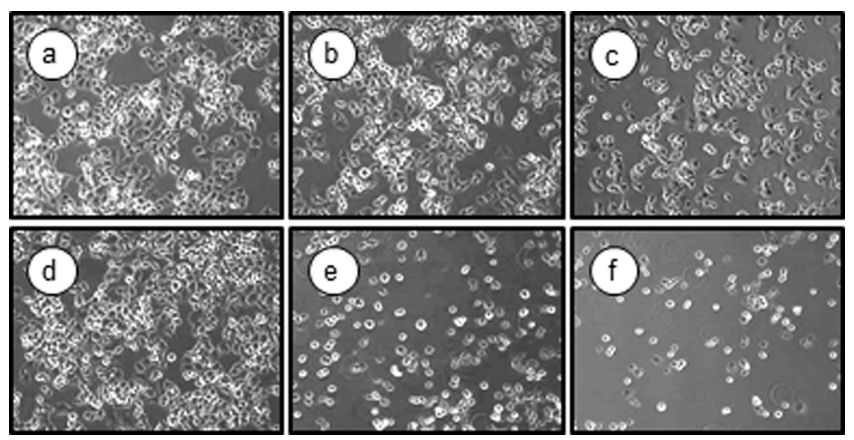

Fig. 2. PST-A and B induced morphological changes in glucose deprived PANC-1 cells. The cells were exposed to the indicated $10 \mu \mathrm{M}$ of PST-A and B under normal and glucose deprivation conditions. PANC-1 cells were exposed to the PST$\mathrm{A}$ and $\mathrm{B}$ for $24 \mathrm{~h}$ in the present or absent of 2DG. PANC-1 cells indicated highly selective cytotoxicity during 2DG (20 mM) stress conditions (a, normal-control; b, normal-10 $\mu$ M PST-A; c, normal-10 $\mu$ M PST-B; d, 2DG-control; e, 2DG-10 $\mu$ M PST-A; f, 2DG-10 $\mu$ M PST-B). Photographs were taken at 100× magnification.

proteins were resolved on 4 20\% gel using SDS-PAGE and transferred onto an immunoblot PVDF membrane (Bio-Rad, USA) for western blotting. Nitrocellulose membranes containing the transferred proteins were blocked in Tris-buffered saline containing 5\% non-fat dry skim milk and $0.1 \%$ Tween 20 for $1 \mathrm{~h}$ at room temperature. Membranes were probed with anti-KDEL mouse monoclonal antibody (for detection of GRP78; StressGen, Victoria, British Columbia, Canada) at 1:500 and anti- $\beta$-Actin mouse monoclonal antibody (Sigma) at 1:1000. Anti-mouse IgG HRP (Amersham Pharmacia Biotech, Japan) at 1:500 was used as a secondary antibody. $\beta$-actin protein was used as the loading control for data normalization. The blots were developed using the enhanced chemiluminescence detection kit (Amersham Pharmacia Biotech) according to the manufacturer's instructions.

\section{Hoechst Staining}

The morphological change characteristic apoptosis were investigated by staining the cells with Hoechst 33342 (Sigma). PANC-1 cells $\left(2 \times 10^{5} \mathrm{cells} / \mathrm{ml}\right)$ were then washed with phosphate-buffered saline (PBS) and fixed in PBS containing $10 \%$ formaldehyde for $4 \mathrm{~h}$ at room temperature. Fixed cells were washed with PBS, and stained with Hoechst $33342(10 \mu \mathrm{M})$ solution at room temperature in the dark for $30 \mathrm{~min}$. The cells were washed twice more with PBS and the hoechst-stained nuclei were visualized by using a fluorescence microscope (TS 100-F; Nikon). Photographs were taken at $400 \times$ magnification.

\section{Statistical Analysis}

All data presented were the means as a standard deviation of three determinations. Data were analyzed using the SPSS package for Windows (Version 14.0; USA) and evaluated by one-way analysis of variance (ANOVA) followed by Scheffe's test. The differences were considered significant at $p<0.05$.

\section{Results}

Selective Cytotoxic Effect of PST-A and B on the Glucose-deprived PANC-1 Cells

To investigate whether PST-A and B have a selective cytotoxicity in glucose-deprived PANC-1 human pancreatic cancer cells, cells were pretreated with various concentrations of PST-A and B in the presence or absence of 2DG. The cytotoxic effects of PST-A and B on PANC-1 morphological alterations were determined by phase-contrast microscopy. Fig. 2 shows PANC-1 cells exposed to $10 \mu \mathrm{M}$ of PST-A and B under the presence or
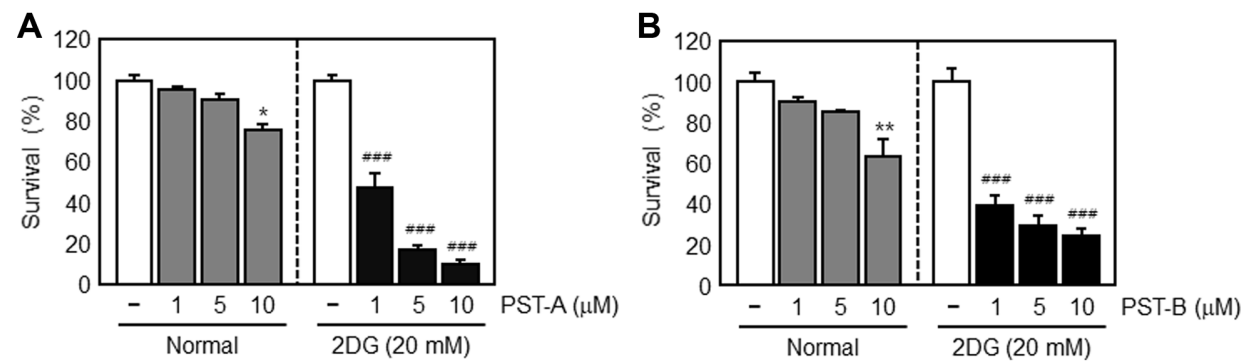

Fig. 3. Selective cytotoxicity of PST-A and B under normal and glucose deprivation. Colony formation analysis of PANC-1 cells exposed to the indicated concentration of PST-A and B for $24 \mathrm{~h}$ in the presence and the absence of $2 \mathrm{DG}$, the survival rate was calculated by setting each of the control survival rates. After 7-8 days, the formed colonies were fixed with $10 \%$ formaldehyde, stained with $0.01 \%$ crystal violet, and counted. ${ }^{*} p<0.05 ;{ }^{* *} p<0.01$ compared with normal growth control. ${ }^{\# \# \#} p<$ 0.001 compared with glucose deprivation control. 
absence 2DG. PST-A and B exhibited no cytotoxicity under normal growth conditions. However, cells exhibited cytoplasmic shrinkage and were either detached from each other or floated in the medium under 2DG stress condition.

We then examined the specific cytotoxic effect of PST-A and B on PANC-1 cells under glucose-deprived conditions by using the colony formation assay. Under normal growth conditions, PST-A and B treatment of PANC- 1 cells had only a weak effect on cell viability. In contrast, cells exposed to PST-A and B at 1, 5, and $10 \mu \mathrm{M}$ showed increase in cytotoxicity as compared to the normal growth controls (Figs. 3A and 3B). Therefore, these results indicate that PST-A and B are not cytotoxic to PANC-1 cells under normal growth conditions. However, PST-A and B treatment led to reduced pancreatic cancer cell viability under glucose deprivation.

\section{Effect of PST-A and B on GRP78 Protein Expression in Glucose-deprived PANC-1 Cells}

Glucose deprivation is a physiological cell condition that leads to accumulation of unfolded or misfolded proteins in the ER lumen of eukaryotic cells. The increase in the unfolded proteins initiates the UPR. The transcriptional activation of GRP78 is the hallmark of UPR that plays a critical role in tumor development, progression, and resistance to chemotherapy [18]. Up-regulation of GRP78 appears to promote tumor growth and progression as well as drug resistance. Agents targeting the UPR pathway may provide a novel therapeutic strategy against cancer. GRP78 is induced during glucose deprivation as part of the UPR, which protects cells from apoptosis under glucose-deprived conditions [19]. Therefore, these results were interested in whether PST-A and $\mathrm{B}$ affect GRP78 induction caused by glucose-deprived conditions. Since GPR78 induction constitutes one of the key pro-survival mechanisms of cancer cells in response to glucose deprivation, PST-A and B-induced suppression of GRP78 expression may result in cell death. This assumption was supported by the observed correlation between the suppressed expression of GRP78 by PST-A and B and cytotoxicity.

To determine the potential GRP78 inhibitory activity of PST-A and B in glucose deprived conditions, the GRP78 protein was performed western blot analysis of the protein samples obtained from PANC-1 cells that had been subjected to glucose deprivation for $24 \mathrm{~h}$ in the presence or absence of PST-A and B. PST-A and B treatment resulted in the down-regulation of stress-induced GRP78 protein expression in a dose-dependent manner in the 2DG-treated PANC-1 cells. However, no such effect on the expression level of GRP78 was observed under normal growth conditions. These results provide evidence that PST-A and B suppress the GRP78 expression induced by glucose-deprived conditions as observed by the inhibition of tumor growth in glucose deprived PANC-1 cells (Figs. 4A and 4B). Suppression of GRP78 expression in glucose deprived conditions leads to reduced cell survival and increased apoptosis. Thus, PST-A and B may be good candidate to explore potential cancer therapy.

\section{PST-A and B Induce the Apoptotic Response in Glucose-deprived PANC-1 Cells}

Apoptosis is the mechanism by which cells undergo programmed cell death to balance cell proliferation or respond to DNA damage [20]. It is characterized by membrane blebbing, shrinking of the cytoplasm, nuclear fragmentation, and the formation of distinct apoptotic bodies [21]. Apoptosis plays an important role in maintenance of tissue homeostasis and protects against carcinogenesis by eliminating damaged cells or abnormal excess cells $[22,23]$. The understanding of apoptosis provided the basis for targeted therapies, and many chemotherapeutic agents exert their anticancer effects by inducing apoptosis in cancer cells. Therefore, induction of apoptosis is relevant to understanding cancer and developing more effective anticancer therapy [24].

To investigate the mechanism of the cell death induced by PST-A and B, examined the apoptotic activity of PANC-1 cells in response to PST-A and B treatment under glucose deprivation conditions. I investigated several hallmarks of apoptosis, such as nuclear chromatin condensation and fragmentation of DNA using Hoechst 33342 staining. Apoptotic cells was collected and counted after PST-A and B treatment in the presence or absence of 2DG

\section{A}

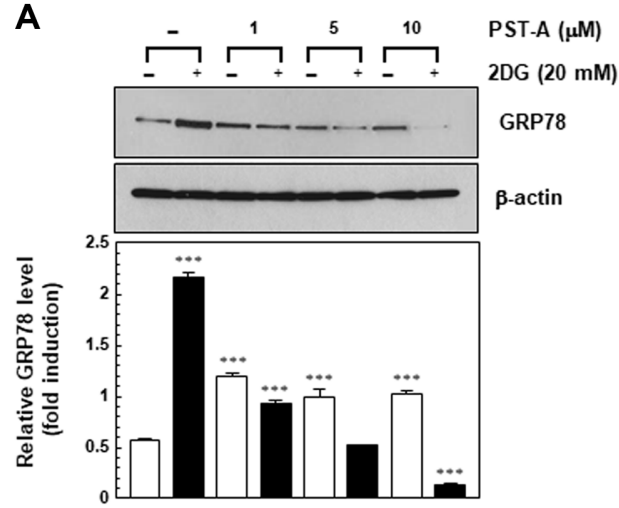

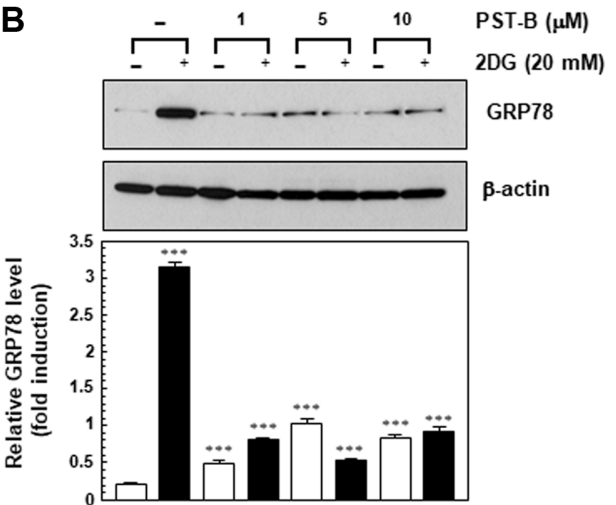

Fig. 4. PST-A and B induced the down-regulation of GRP78 in the PANC-1 cells. Total cell lysates of PANC-1 cells were prepared and subjected to western blot analysis using mouse monoclonal anti-KDEL antibody. PNAC-1 cells were treated with PST-A and B indicated concentration for $24 \mathrm{~h}$ under $2 \mathrm{DG}(20 \mathrm{mM})$ conditions. $\beta$-actin was measured as an internal loading control. ${ }^{* *} p<0.001$ compared with normal growth control. 

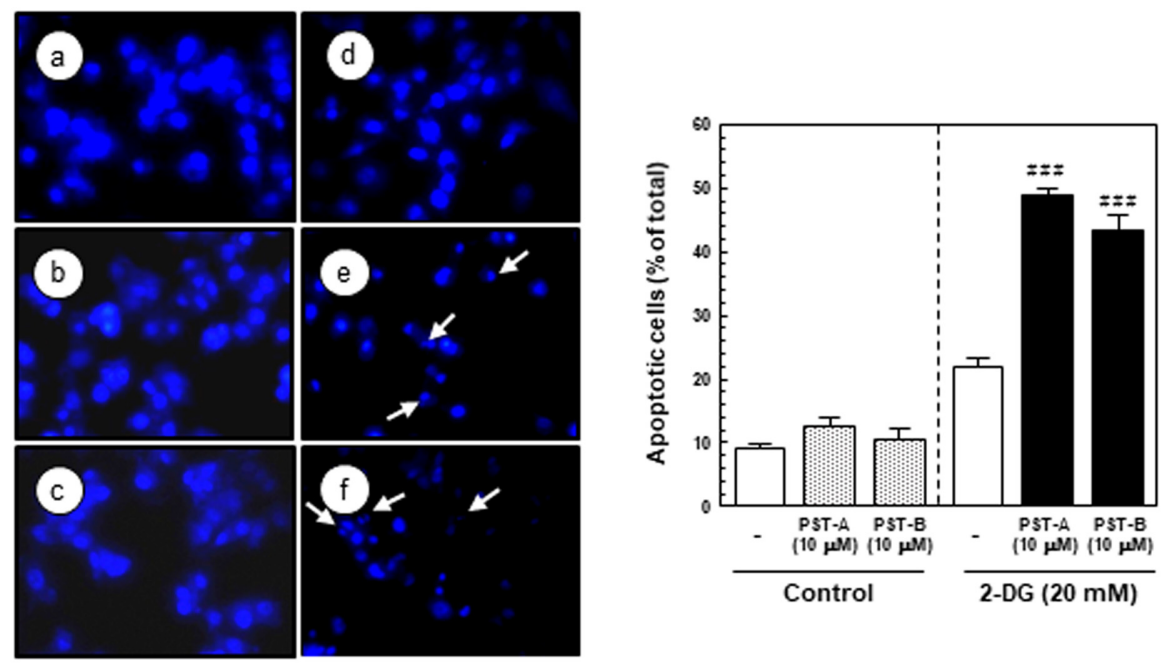

Fig. 5. PST-A and B induced apoptosis under 2DG stress conditions. The cells were exposed to the indicated $10 \mu \mathrm{M}$ of PST-A and B under normal and glucose deprivation condition. PANC- 1 cells were exposed to the PST-A and B for $24 \mathrm{~h}$ in the present or absent of 2DG. (A) Apoptosis nuclear and condensation and fragmentation induced by PST-A and B on contained 2DG PANC-1 cells (a, normal-control; b, normal-10 $\mu$ M PST-A; c, normal-10 $\mu$ M PST-B; d, 2DG-control; e, 2DG-10 $\mu$ M PSTA; f, 2DG-10 $\mu$ M PST-B). Photographs were taken using a blue filter at a magnification of $\times 400$. Arrow indicates an apoptotic cell with apoptotic bodies. (B) This histogram represents the percentage of apoptotic PANC-1 cells in total cell population after different treatment. ${ }^{\# \# \#} p<0.001$ compared with glucose deprivation control.

and examined the morphological changes in their nuclei. Pancreatic cancer cells treated with $10 \mu \mathrm{M}$ of PST-A and $B$ showed marked chromatin condensation and the formation of apoptotic bodies in the cell group exposed to 2DG, but no such observation was made for the cell group that was not exposed to 2DG (Fig. 5).

\section{Discussion}

Solid tumors, especially pancreatic cancer, are surrounded by stressful microenvironments, including glucose deprivation, hypoxia and nutrient deprivation [25]. These microenvironmental conditions, especially glucose deprivation, activate the UPR, a stress-signaling pathway, in tumor cells [26, 27]. Tumor cells need to adapt to the microenvironmental conditions and induction of UPR is an important system in this response. GRP78 binds to the ER transmembrane sensor proteins and maintains them in an inactive form. The accumulation of unfolded proteins in the ER activates the UPR, which enhances cell survival by induction of GRP78 [28,29]. Several reports have demonstrated that GRP78 plays a role in protecting tumor cells against the toxic effects of anticancer agents [12-14]. GRP78 has been established to be important for tumor progression and chemotherapeutic drug resistance $[30,31]$. Induction of GRP78 expression in solid tumors protects tumor cells against glucose deprivation. Therefore, GRP78 proteins in glucose-deprived cells may be a potent therapeutic target for anticancer therapy [32].

In this study have demonstrated that the PST-A and B have selective cytotoxicity on pancreatic cancer cells under 2DG-induced glucose deprivation. 2DG is a synthetic analogue of glucose in which the hydroxyl group at the second position carbon is replaced by hydrogen, and causes a block in glycolysis and glycosylation [33]. 2DG also induces UPR in the ER due to low glucose stress [34]. I isolated novel compounds from Ponciri Fructus, designated pancastatin A (PST-A) and B (PST-B). Ponciri Fructus has previously been reported to induce antiinflammatory and anti-helicobacter pylori effect [35]. Specifically, PST-A and B inhibited the expression of the UPR target gene, GRP78 during glucose deprivation. Disruption of the UPR may therefore, provide a novel therapeutic approach to targeting glucose-deprived solid tumors.

This study indicates that PST-A and B can be potential candidates and a novel therapeutic approach to induce selective cell death under glucose deprivation in pancreatic cancer cells. As shown in Figs. 2 and 3, PST-A and B had an inhibitory effect on tumor cell viability of pancreatic cancer cells under glucose-deprived conditions. Expectedly, they had no effect under normal growth conditions. Moreover, as shown in Fig. 5, PST-A and B lead to apoptosis. PANC-1 cells treated with PST-A and B showed chromatin condensation and the formation of apoptotic bodies in the group to 2DG. On the assumption that, verified mechanisms of the PST-A and B connoted these selective killing activities. As expected, western blotting confirmed that PST-A and B significantly decreases the 2DG induced GRP78 protein levels in PANC-1 cells (Fig. 4).

In conclusion, PST-A and B had no cytotoxic effect under normal growth conditions, but showed selective cytotoxicity against PANC-1 cells under glucose deprivation. This study indicated that PST-A and B induces GRP78 down-regulation in glucose deprived PANC-1 pancreatic cancer cells. These results indicate that PST-A and $\mathrm{B}$ may be potential novel therapeutic candidates to induce selective cell death under glucose deprivation in pancreatic cancer cells. 


\section{Acknowledgments}

This work was supported by Kyungnam University Foundation Grant, 2016.

\section{Conflict of Interest}

The authors have no financial conflicts of interest to declare.

\section{References}

1. Jemal A, Thomas A, Murray T, Thun M. 2002. Cancer statistics. CA: Cancer J, Clin. 52: 23-47.

2. Chang DK, Merrett ND, Biankin AV. 2008. Pancreatic Cancer Network, Improving outcomes for operable pancreatic cancer: is access to safer surgery the problem? J. Gastroenterol. Hepatol. 23: 1036-1045.

3. Welsch T, Degrate L, Zschabitz S, Hofer S, Werner J. 2011. The need for extended intensive care after pancreaticoduodenectomy for pancreatic ductal adenocarcinoma. Langenbecks Arch. Surg. 396: 353-362.

4. Guha S, Lunn JA, Santiskulvong C, Rozengurt E. 2003. Neurotensin stimulates protein kinase C-dependent mitogenic signaling in human pancreatic carcinoma cell line PANC-1. Cancer Res. 63: 2379-2387.

5. Kokkinakis DM, Liu XY, Neuner RD. 2005. Modulation of cell cycle and gene expression in pancreatic tumor cell lines by methionine deprivation (methionine stress): implications to the therapy of pancreatic adenocarcinoma. Mol. Cancer Ther. 4: 1338-1348.

6. Awale S, Lu J, Kalauni SK, Kurashima Y, Tezuka Y, Kadota S, Esumi, H. 2006. Identification of arctigenin as an antitumor agent having the ability to eliminate the tolerance of cancer cells to nutrient starvation. Cancer Res. 66: 1751-1757.

7. Izuishi K, Kato K, Ogura T, Kinoshita T, Esumi H. 2000. Remarkable tolerance of tumor cells to nutrient deprivation: possible new biochemical target for cancer therapy. Cancer Res. 60, 6201-6207.

8. Ogiso Y, Tomida A, Lei S, Omura S, Tsuruo T. 2000. Proteasome inhibition circumvents solid tumor resistance to topoisomerase IIdirected drug. Cancer Res. 60: 2429-2434.

9. Semenza GL. 2003. Angiogenesis in ischemic and neoplastic disorders. Annu. Rev. Med. 54: 17-28.

10. Luo S, Baumeister P, Yang S, Abcouwer SF, Lee AS. 2003. Induction of Grp78/BiP by translational block: activation of the Grp78 promoter by ATF4 through and upstream ATF/CRE site independent of the endoplasmic reticulum stress elements. J. Biol. Chem. 278: $37375-37385$.

11. Park HR, Tomida A, Sato S, Tsukumo Y, Yun J, Yamori T, et al. 2004. Effect on tumor cells of blocking survival response to glucose deprivation. J. Natl. Cancer Inst. 17: 1300-1310.

12. Park HR, Ryoo IJ, Choo SJ, Hwang JH, Kim JY, Cha MR, et al. 2007. Glucose-deprivated HT-29 human colon carcinoma cells are sensitive to verrucosidin as a GRP78 down-regulator. Toxicology 229: 253-261.

13. Hetz C, Bernasconi P, Fisher J, Lee AH, Bassik MC, Antocsson B, et al. 2006. Proapoptotic BAX and BAK modulate the unfolded protein response by a direct interaction with IRE1a. Science 312: 572-576.

14. Li J, Lee AS. 2006. Stress induction of GRP/BiP and its role in cancer. Curr. Mol. Med. 6: 45-54.

15. Zhang K, Kaufman RJ. 2004. Signaling the unfolded protein response from the Endoplasmic reticulum. J. Biol. Chem. 279: 2593525938.

16. Garber K. 2006. Researchers target unfolded protein response in cancerous tumor growth. J. Natl. Cancer Inst. 98: $152-154$.

17. Choi AR, Lee IK, Woo EE, Kwon JW, Yun BS, Park HR. 2015. New glabretal triterpenes from the immature fruits of Poncirus trifoliate and their selective cytotoxicity. Chem. Pharm. Bull. 63: 1065-1069.

18. Lee AS. 2007. GRP78 induction in cancer: therapeutic and prognostic implications. Cancer Res. 67: 3496-3499.

19. Fu Y, Lee AS. 2006. Glucose regulated proteins in cancer progression, drug resistance and immunotherapy. Cancer Biol. Ther. 5: 741744

20. Tsujimoto Y, Cossman J, Jaffe E, Croce CM. 1985. Involvement of the bcl-2 gene in human follicular lymphoma. Science 228: 14401443.

21. Hu W, Kavanagh JJ. 2003. Anticancer therapy targeting the apoptotic pathway. Lancet Oncol. 4: 721-729.

22. Kaufmann SH, Hengartner MO. 2001. Programmed cell death: alive and well in the new millennium. Trends Cell Biol. 11: 526-534.

23. Jin Z, El-Deiry WS. 2005. Overview of cell death signaling pathways. Cancer Biol. Ther. 4: 139-163.

24. Ghobrial IM, Witzig TE, Adjei AA. 2005. Targeting apoptosis pathways in cancer therapy. CA: Cancer J, Clin. 55: 178-194.

25. Kozutsumi Y, Segal M, Normington K, Gething MJ, Sambrook J. 1988. The presence of malfolded proteins in the endoplasmic reticulum signals the induction of glucose-regulated proteins. Nature 332: 462-464.

26. Kaufman RJ. 1999. Stress signalling from the lumen of the endoplasmic reticulum: coordination of gene transcriptional and translational controls. Genes Dev. 13: 1211-1233.

27. Sausville EA. 2004. Versipelostatin: unfolding an unsweetened death. J. Natl. Cancer Inst. 96: 1266-1267.

28. Yun J, Tomida A, Nagata K, Tsuruo T. 1995. Glucose-regulated stresses confer resistnace to VP-16 in human cancer cells through a decreased expression of DNA topoisomerase II. Oncol. Res. 7: 583-590.

29. Tomida A, Tsuruo T. 1999. Drug resistance mediated by cellular stress response to the microenvironment of solid tumors. Anticancer Drug Des. 14: 169-177.

30. Gazit G, Lu J, Lee AS. 1999. De-regulation of GRP stress protein expression in human breast cancer cell line. Breast Cancer Res. Treat. 54: 135-146.

31. Reddy RK, Mao C, Baumeister P, Austin RC, Kaufman RJ, Lee AS. 2003. Endoplasmic reticulum chaperone protein GRP78 protects cells from apoptosis induced by topoisomerase inhibitor. J. Biol. Chem. 278: 20915-20924

32. Kaufman RJ. 2002. Orchestrating the unfolded protein response in health and disease. J. Clin. Invest. 110: 1389-1398.

33. Little E, Ramakrishnan M, Roy B, Gazit G, Lee AS. 1994. The glucose-regulated proteins (GRP78 and GRP94): functions, gene regulation, and applications. Crit. Rev. Eukaryot. Gene Expr. 4: 1-18.

34. Kang HT, Hwang ES. 2006. 2-Deoxyglucsoe: An anticancer and antiviral therapeutic, but not anymore a low glucose mimetic. Life Sci. 78: 1392-1399.

35. Kim DH, Bae EA, Han MJ. 1999. Anti-Helicobacter pylori activity of the metabolites of poncirin from Poncirus trifoliata by human intestinal bacteria. Biol. Pharm. Bull. 22: 422-424. 